\section{SYNAPTIC PHYSIOLOGY}

\section{Speedy synapses}

Although most neuronal synapses operate in an 'all-or-nothing' fashion, some - for example, in the vertebrate retina or the insect ocellus - transmit graded signals. A new study by Peter Simmons, published in Neuron, describes how such transmission at one type of synapse shows an unprecedented dependency on the rate at which the presynaptic neuron is depolarized, rather than on the amplitude of the presynaptic depolarization.

The study focused on three large second-order neurons in the locust ocellar system, which is thought to be used to maintain stability during flight by providing rapid information about changes in the position of the horizon. The neurons - called L1-L3 - form reciprocal inhibitory synapses that produce graded postsynaptic depolarizations, but transmit only transiently, regardless of the duration of presynaptic depolarization.

Simmons found that, although spikes of different amplitudes in the presynaptic neuron produced inhibitory postsynaptic potentials (IPSPs) of different amplitudes in the postsynaptic cell, the size of the presynaptic depolarization was not the determining factor. He used a voltage clamp to depolarize the presynaptic neuron at different rates, and found that the rate of depolarization determined how fast the IPSP developed, and that each IPSP peaked at a fixed time after it had begun. So, because the IPSP can grow for only a limited time and because the rate of growth depends on the rate of presynaptic depolarization, the amplitude of the IPSP is dictated by the rate at which the presynaptic neuron depolarizes. The reason for this behaviour is that the synapse depresses extremely rapidly. By measuring inhibitory postsynaptic currents under voltage clamp, Simmons found that transmitter was released at the synapse for a maximum of $2.1 \mathrm{~ms}$. No other synapse has been found that depresses so rapidly and completely. When the ocellus is operating naturally, rapid decreases in light intensity produce fast rebound spikes, which would be rapid enough to produce IPSPs in the L neurons. These IPSPs might lead to synchrony of spiking in the three neurons. Simmons proposes that this mechanism allows the ocellar system to provide accurate information about the timing of changes in ocellar illumination, allowing the insect to monitor the horizon and maintain flight stability.

\title{
AXON GUIDANCE
}

\section{Taking the easy route}

When peripheral motor axons are damaged, they have what seems to be a remarkable ability to regenerate and reconnect with the muscle target that they innervated before injury. In fact, as Nguyen et al. show in a new paper in Nature Neuroscience, the reinnervation process is even more accurate than was previously thought, and many axons actually re-establish contact with their original neuromuscular junction (NMJ). However, the explanation for this phenomenon is not as complex as one might imagine - the results of this study indicate that the axons simply retrace their original path.

The authors used a mouse line in which a fraction of motor axons expressed yellow or

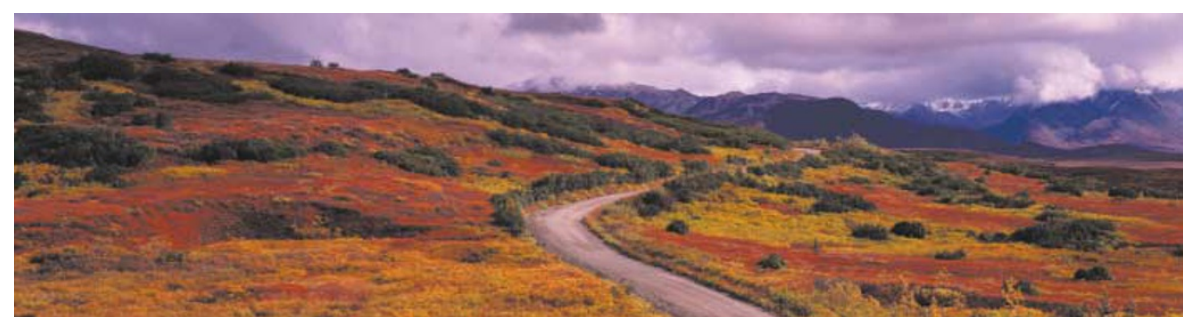

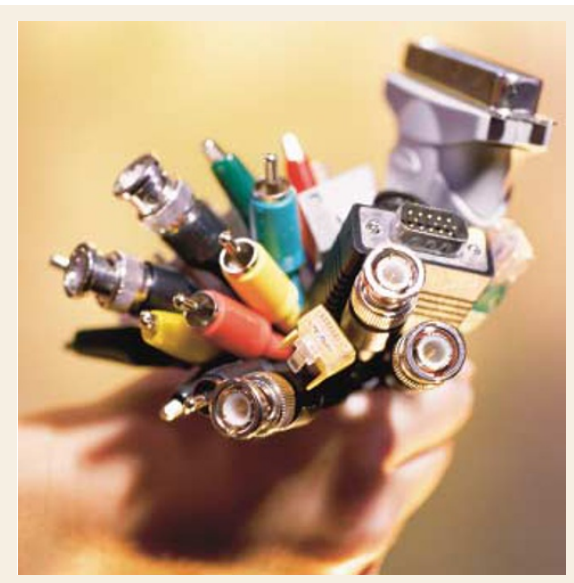

The mechanism of the rapid depression of these synapses is unclear, but it might involve a reduction in the sensitivity of presynaptic vesicle release to calcium entry, as has been found at other invertebrate synapses. Further studies of the dynamics of graded synaptic transmission might lead to similar insights into how these synapses are specialized to function in specific behavioural contexts, such as vision.

Rachel Jones

(4) References and links

ORIGINAL RESEARCH PAPER Simmons, P. J. Presynaptic depolarization rate controls transmission at an invertebrate synapse. Neuron 35, 749-758 (2002)

that the endoneurial tubes — cylindrical arrays of non-neuronal cells that surround the nerve fibres - remain intact. If the nerve is cut, on the other hand, the endoneurial tubes are damaged, and the re-targeting of axons is much less accurate. This indicates that intact endoneurial tubes are required for axon guidance during repair. Also, Nguyen et al. showed that, on the rare occasions when an axon entered the wrong pathway, it followed exactly the same route as the axon that was present before injury, indicating that the guidance cues are not axon-specific.

The authors suggest that a nonspecific mechanical cue might be important for axon guidance during repair. Rather than following a complex array of specific molecular cues, the axons simply grow down a pre-existing tubular network. As recent studies on axon guidance have tended to focus on molecular mechanisms, it is possible that the importance of mechanical cues has been underestimated, and this study by Nguyen et al. goes some way towards redressing the balance.

Heather Wood

(4) References and links

ORIGINAL RESEARCH PAPER Nguyen, Q. T. et al. Pre-existing pathways promote precise projection patterns. Nature Neurosci. 5, 861-867 (2002)

FURTHER READING Fournier, A. E. \& Strittmatter, S. M. Regenerating nerves follow the road more traveled. Nature Neurosci. 5, 821-822 (2002) 